\title{
Das «Berner Modell» - Geographielehrerausbildung und -fortbildung am Geographischen Institut der Universität Bern
}

Mit der Wahl von PD Dr. Eduard Petri zum außerordentlichen Professor für Geographie schuf der Regierungsrat des Kantons Bern am 13. März 1886 das Geographische Institut der Universität Bern. Im Ernennungsbrief vom 19. März 1886 schrieb die Erziehungsdirektion dem Neugewählten, er habe «auch speziell für die Lehreramtskandidaten Vorlesungen über die Geographie» abzuhalten (STAB Universitätsakten 1886; vgl. GROSJEAN 1986). Die Lehrerausbildung und die Unterstützung der Schulgeographie gehören damit zu den Grundaufgaben des Geographischen Institutes und seien im folgenden unter der Vielzahl der heutigen Tätigkeitsgebiete des Institutes herausgegriffen.

\section{Die Ausbildung von Geographielehrern als Aufgabe des Institutes}

Die Lehrerausbildung und -weiterbildung ist zunächst in die generelle Aufgabe eines Universitätsinstitutes einzuordnen.

Die Universitätsinstitute sind zu Forschung, Lehre und in einem unterschiedlichen Ausmaß zur Übernahme von Dienstleistungen zuhanden der Öffentlichkeit verpflichtet. Um diese Aufgabe zu erfüllen, haben die Wissenschafter «Wissen zu schaffen» und in der Lehre zweckmäßig aufbereitet (Umsetzung)

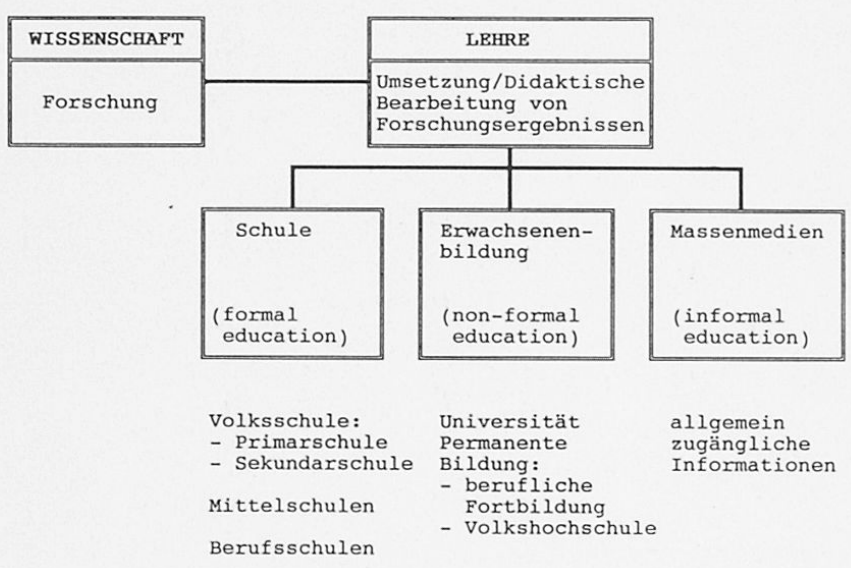

Entwurf: K. Aerni

Abb. 1 Die Forschung und die drei Stufen der Umsetzung/ Erziehung weiterzugeben (Abb. 1). Das heißt, daß die wissenschaftliche Tätigkeit nur die erste Phase der Arbeit ist. Wissenschaftliche Ergebnisse dürfen nicht nur zur Fortführung des Forschungsprozesses erarbeitet und publiziert werden, um dann nach Erfüllung der Funktion als Selbstzweck in Schubladen und Archiven $z u$ verschwinden. Die wissenschaftlich orientierten Publikationen und Ergebnisse der ersten Arbeitsphase sind in einer zweiten Phase in allgemein verständliche Informationen umzusetzen - und da wird das Problem schwieriger. Was wir erreichen müssen, ist die Ausnutzung der Forschungsergebnisse als Informationsquellen, die der Gesellschaft zur Klärung von Problemen dienen, sie zum Nachdenken veranlassen und ihr Anhaltspunkte geben, ihr Verhalten kritisch im Hinblick auf die Zukunft zu reflektieren. Hier liegt die Bedeutung der Umsetzung der Forschungsergebnisse in die Lehre, in die Medien und in Aktivitäten, wie sie etwa von der Volkshochschule und den Geographischen Gesellschaften wahrgenommen werden.

Die Informationsbedürfnisse der Gesellschaft ändern sich mit ihrer Struktur. Der Übergang von der Industriegesellschaft zur Dienstleistungsgesellschaft brachte einerseits mit der Reduktion der Arbeitszeit ein erhöhtes Informationsbedürfnis und förderte andererseits durch das rasch wachsende Wissen die Erwachsenenbildung. Damit haben sich die traditionellen Bildungsstrukturen differenziert, und wir unterscheiden folgende Typen (VERDUINMULLER 1983: 9-2, vgl. Abb. 1):

1. Das Schulsystem klassischer Prägung mit der gesetzlich vorgeschriebenen Volksschule, gefolgt von der Berufsschule oder Mittelschule.

2. Die Erwachsenenbildung in den Formen der universitären Bildung, des zweiten Bildungsweges und der permanenten Bildung. In Anbetracht der abgesunkenen Schülerzahlen und des steigenden Bildungsbedürfnisses der Erwachsenen muß sich die Lehrerbildung zunehmend auf die Erwachsenen ausrichten.

Klaus Aerni, Prof. Dr.; Martin Hasler, Dr., Lehrbeauftragter für Fachdidaktik, Geogr. Institut Universität Bern, Hallerstr. 12, 3012 Bern 
3. Die allgemeine Information, ausgehend von den Massenmedien und aufgenommen vom Einzelmenschen. Diese Art der Bildung erreicht heute eine zunehmend umweltbewußte Bevölkerung, die nach mehr Informationen verlangt und durch Bürgerinitiativen auf Planung und Gestaltung unseres Lebensraumes Einfluß nehmen will.

Zusammenfassend ergibt sich, daß Lehre und Forschung gleichgewichtig sind und daß unser «didaktisches Umfeld» die Schulen aller Stufen, die Erwachsenenbildung und die allgemeine Information der Gesellschaft zu umfassen hat. Die Schweizer Geographen haben sich in den Grundsatzdiskussionen der Jahre 1986/87 positiv zu dieser Verpflichtung bekannt (AERNI 1986, 1988; LEIMGRUBER 1988, VSGg 1986)

\section{Die Verknüpfung der Lehrerausbildung mit dem Institut - das «Berner Modell»}

Die Arbeit des Geographischen Institutes besteht aus den Bereichen Forschung, Lehre und Umsetzung (Abb. 2). Die Forschung spielt sich in acht Forschungsgruppen ab, die vielseitige Kontakte zu nationalen und internationalen Instituten besitzen. Die Forschungsgruppen sind aus Gründen der Organisation und für die Bedürfnisse der Lehre in die beiden Abteilungen «Physische Geographie und Kulturgeographie» zusammengefaßt. Nach dem Abschluß der Studien durch das Lizentiatsexamen stehen den Geographen drei Möglichkeiten offen:

a) Einstieg ins Berufsleben als Geograph

b) Berufsausbildung zum Gymnasiallehrer

c) Doktoratsstudium

Die Ausbildung der Geographielehrer an der Universität Bern erfolgt nicht an einer höheren pädagogischen Abteilung, sondern am Geographischen Institut.

Für die SekundarstufeI (Sekundarschule, 5. bis 9. Schuljahr) regelt die Institution des «Sekundarlehramtes» (SLA) das Studium. Die fachspezifische Ausbildung der Studenten erfolgt an den Instituten und Seminaren der phil.-hist. und phil.-nat. Fakultät. Am Geographischen Institut in Bern absolvieren die Studenten des SLA im Prinzip das Grundstudium der Fakultätsstudenten, erstreckt auf 6 Semester. Dabei tritt an die Stelle der quantitativen Geographie ein Kurs zur Analyse/Synthese der Dynamik des Landschaftswandels an schweizerischen Landschaftstypen sowie eine Feldarbeit (Hausarbeit). Die Studien am SLA können für ein Weiterstudium angerechnet werden.

Die Fachdidaktik Geographie wird als einsemestriger Kurs von amtierenden Sekundarlehrern durchgeführt.

Die Ausbildung zukünftiger Lehrer der Sekundarstufe II (10.-14. Schuljahr) wird in der Verordnung für das Höhere Lehramt (VHL) umschrieben. Verlangt werden zum Erwerb des Diploms für das Höhere Lehramt einerseits ein Fachdiplom und andererseits die Absolvierung einer didaktisch-erziehungswissenschaftlichen Ausbildung (Abb. 3). Ein zukünftiger Geographielehrer an einer Mittelschule durchläuft also ein Geographiestudium, das er mit dem Diplom abschließt. Zudem studiert er in einem zweiten (evtl. dritten) Fach noch bis zum Zweitfachabschluß. Damit wird die fachliche Qualifikation für den Mittelschulunterricht sichergestellt. Die berufsbezogene Ausbildung verläuft dreistufig und untersteht der Abteilung für das Höhere Lehramt, die nach Absolvierung der Fachstudien und der erziehungswissenschaftlich-didaktischen Ausbildung das Diplom für das Höhere Lehramt ausstellt.

Im «Berner Modell» (Abb. 2) kommt dem Fachdidaktiker in der Ausbildung zukünftiger Mittelschullehrer eine wesentliche Bedeutung zu:

Gewählt durch die Kommission für das Höhere Lehramt - in der Regel werden Mittelschullehrer mit mehrjähriger Unterrichtserfahrung berufen und teilzeitlich angestellt - findet der Geographiedidaktiker seinen Arbeitsplatz am Geographischen Institut.

Zu seinem Tätigkeitsfeld gehören die Durchführung eines zweistündigen Kurses, die Betreuung der Lehrpraktika (Besuche, Studentenberatung, Abnahme von Probelektionen) und von Hausarbeiten sowie die Abnahme von Schlußprüfungen.

Der fachdidaktische Kurs führt zweistündig über zwei Semester (Abb. 3) in die praktische Unterrichtsarbeit ein. Neben der Vermittlung theoretischer Grundlagen stehen praktische Fragen der Unterrichtsvorbereitung und -gestaltung im Vorder-

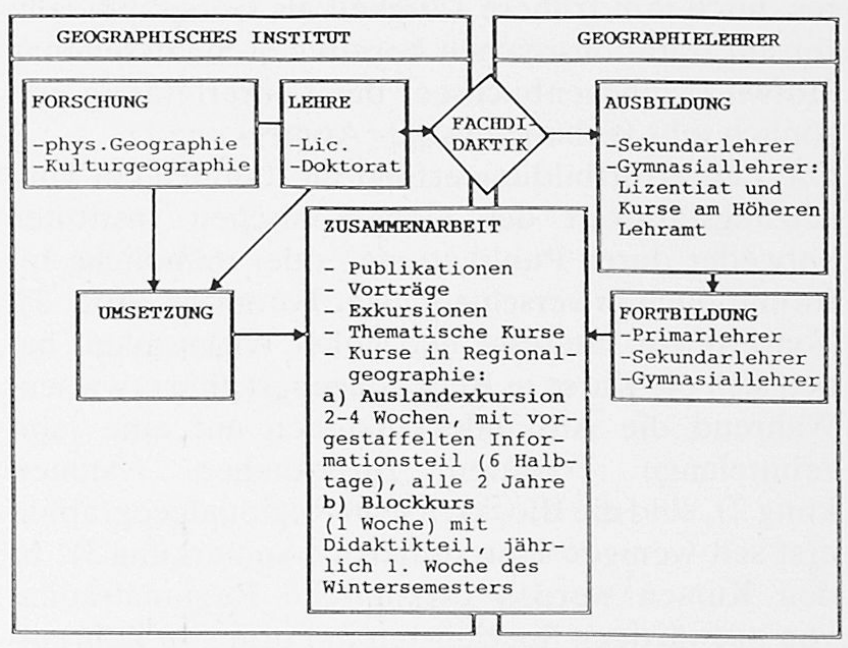

Entwurf: K. Aern

Abb. 2 Das «Berner Modell» für die Aus- und Fortbildung von Geographielehrern 


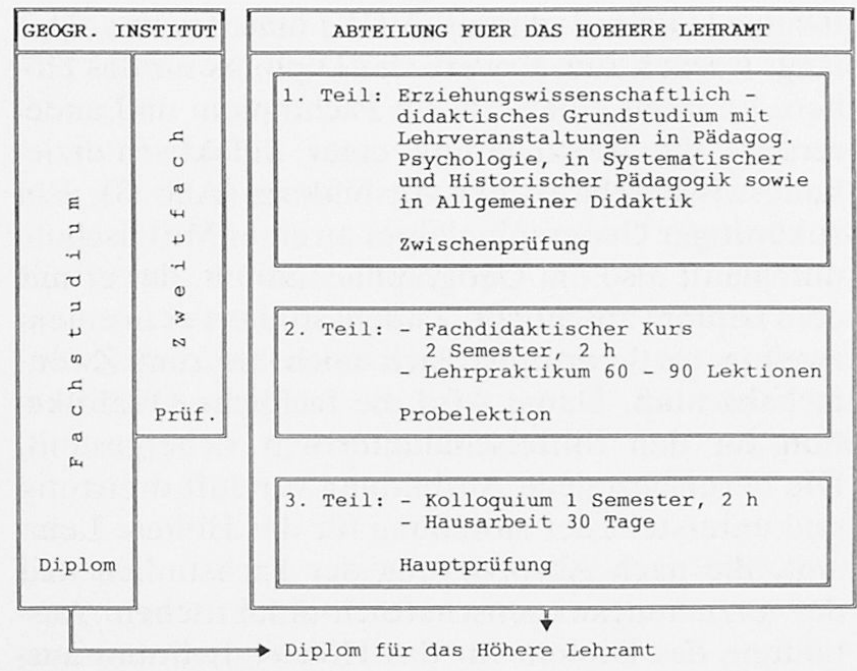

Entwurf: M. Hasler

Abb. 3 Erziehungswissenschaftlich-didaktische Ausbildung am Höheren Lehramt der Universität Bern

grund. Der Kurs soll dem Studenten den Einstieg in das nachfolgende Lehrpraktikum erleichtern.

Enge Kontakte zum VSG (Verein Schweizerischer Gymnasiallehrer) und VSGg (Verein schweizerischer Geographielehrer), die Mitarbeit und Durchführung von Weiterbildungskursen und die Mitarbeit an Publikationen und Kontakte zu Kollegen im In- und Ausland runden das vielfältige Beziehungsnetz des Geographiedidaktikers ab.

Die Fortbildung der Lehrer erfaßt nun nicht bloß die Absolventen des Institutes, sondern kommt dank der ausgezeichneten vertikalen Verbindungen der Dozenten (die meisten waren früher selber Primar-, Sekundar- oder Gymnasiallehrer) den verschiedenen Lehrerorganisationen aller Schulstufen zugute (Abb.2). Von großer Bedeutung ist immer noch, daß eine Reihe von Professoren des Berner Institutes durch ihre frühere Tätigkeit als Geographielehrer am traditionsreichen bernischen Staatsseminar Hofwil (Münchenbuchsee) der Lehrerbildung persönlich sehr verbunden sind (Anmerkung 1).

Die Lehrerfortbildung erfolgt im Rahmen der Umsetzungstätigkeit des Geographischen Institutes entweder durch Publikationen oder mündliche Informationen in verschiedenster Form (vgl. Abb. 2). Von großem Interesse und hoher Wirksamkeit haben sich die Kurse in Regionalgeographie erwiesen. Während die Auslandexkursionen auf eine jahrzehntelange Tradition zurückgehen (Anmerkung 2), sind die Blockkurse in Regionalgeographie erst seit wenigen Jahren üblich (Anmerkung 3). In den Kursen werden dynamische Regionalräume von auswärtigen Spezialisten in Form von Lehraufträgen dargestellt. Die Vorlesungen gehören zum normalen Studienplan und können von allen Studenten sowie amtierenden Lehrern im Rahmen der vom Kanton organisierten Lehrerfortbildung besucht werden. In einem Teil der verfügbaren Zeit (Nachmittag) setzen die Studenten des Höheren Lehramtes und die Lehrer den Kursinhalt unter Leitung des Fachdidaktikers des Institutes um, wodurch die angestrebte Dreieckbeziehung Forschung-Lehre-Umsetzung/Praxis direkt hergestellt wird (vgl. Abb. 2).

\section{Die Publikationen des Geographischen Institutes}

In Form von Publikationen erreichen wissenschaftliche Resultate und deren Umsetzungen am ehesten eine weite Verbreitung. Daher hat sich das Geographische Institut seit jeher um eine gute Zusammenarbeit mit andern Fach- und Amtsstellen und Stiftungen bemüht. Besonders eng waren stets die Kontakte zur Geographischen Gesellschaft von Bern, da stets eine Reihe von Dozenten auch im Vorstand der Gesellschaft tätig waren.

Die Publikationstätigkeit des Institutes wurde 1975 institutionalisiert durch die Gründung der Arbeitsgemeinschaft «Geographica Bernensia», in der sich die Geographische Gesellschaft und die Fachschaft der Geographiestudenten als Kollektivmitglieder mit den habilitierten Dozenten als Einzelmitgliedern verband. Seit 1975 wurden rund 100 Arbeiten publiziert, gegliedert in die Reihen A - African Studies (8 Bände), B - Berichte über Exkursionen, Studienlager und Seminarveranstaltungen (8), G Grundlagenforschung (31), P - Geographie für die Praxis (17), S - Geographie für die Schule (13) und U - Skripten für den Universitätsunterricht (19). Abb. 4 veranschaulicht das Publikationskonzept und zeigt, daß das Institut über eine Großzahl von Partnern in der Produktion sowie über ein weitgespanntes Abnehmernetz verfügt. Zum aktiven Marketing gehört auch die Präsenz der «Geographica Bernensia» an wissenschaftlichen und an schulorientierten Tagungen.

Eine besondere Stärke der Fachdidaktik Geographie am Berner Institut kommt darin zum Ausdruck, daß geographische Unterrichtseinheiten in Form von Lizentiats- oder Doktorarbeiten verfaßt werden können (Abb. 5). Neben der wissenschaftlichen Erarbeitung des Stoffes sind Schulerfahrung (Primar- oder Sekundarlehrerpatent und entsprechende Praxis), genügend Studienzeit und eigene Erprobungsmöglichkeiten des Unterrichtsentwurfes persönliche Voraussetzungen, die nicht von allen Kandidaten erfüllt werden können.

An dieser Stelle seien die Arbeiten von PFANDER (1984), «Exkursionsvorschläge für den Raum des Kantons Bern und Didaktik geographischer Geländearbeit», STUCKI (1984), «Vulkan Dritte Welt/Indonesien» und BUFF (1987), das «Berggebiet», angeführt. Im Prinzip wurde das konzeptionelle Modell 
nach Abb. 5 auch in den zahlreichen Lehrerfortbildungskursen angewandt, die dem Erarbeiten von Unterrichtseinheiten dienten (BINZEGGER u. GRÜTTER 1981, GRÜTTER et al. 1982).

Abschließend kann gesagt werden, daß das Engagement des Geographischen Institutes in der Lehreraus- und fortbildung und im Publizieren von Unterrichtseinheiten sich sehr bewährt. Eingesetzt hat diese Arbeit mit der Entwicklung des Lehrmittels «Die Schweiz und die Welt im Wandel» (AERNI et al. 1979), wozu die Vorarbeiten 1973 eingeleitet worden sind. Das Resultat bewirkte mehrere Fortsetzungsarbeiten in andern Kantonen, führte zu einer Erhöhung der Stundendotation in der Volksschule, konnte in die Lehrplanreform der bernischen Volksschule integriert werden, und das Wichtigste, es animierte zahlreiche Lehrergruppen zur selbständigen Bearbeitung ihrer Region.

\section{Ausblicke}

Auf verschiedenen Ebenen, sowohl bei der Erziehungsdirektion wie auch bei der Universität, werden die zukünftige Bildungslandschaft und die Auf-

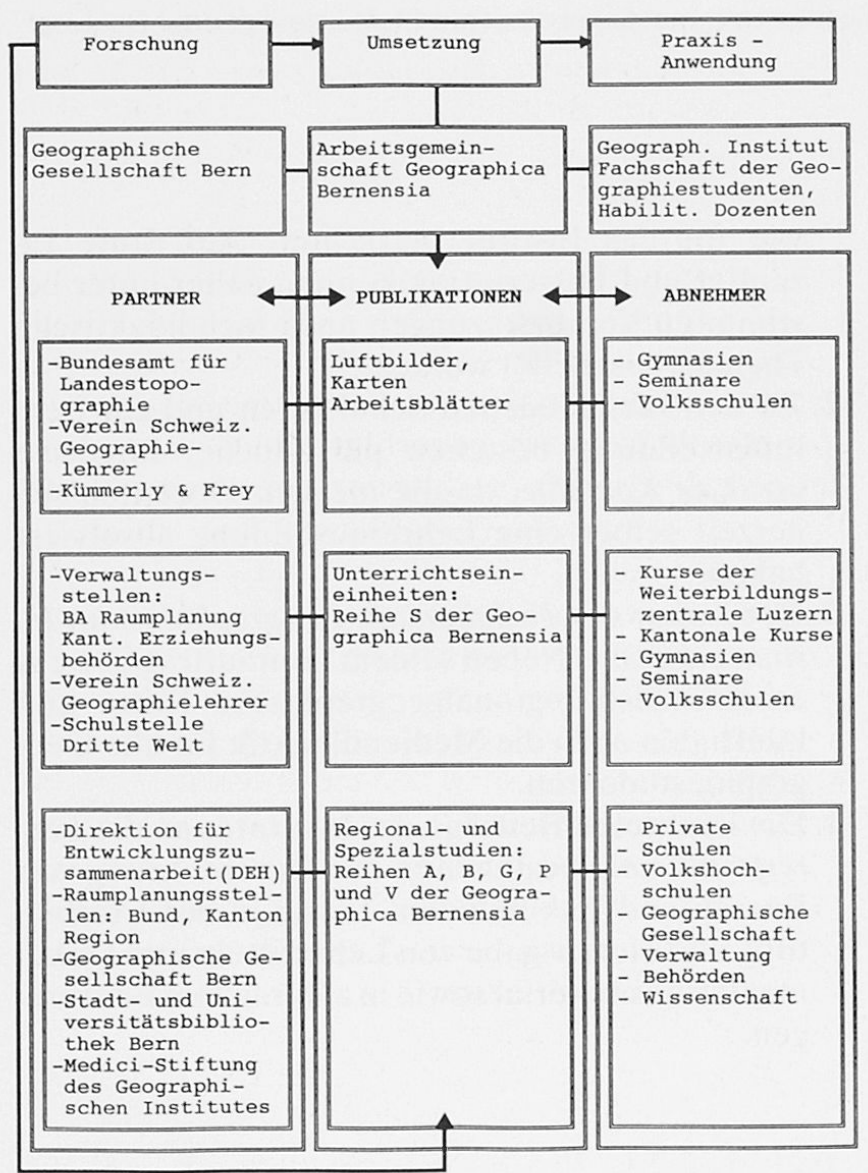

Entwurf: K. Aerni

Abb. 4 Publikationskonzept des Geographischen Institutes der Universität Bern (Schema) gaben der Lehrerbildungsinstitutionen intensiv diskutiert. Strukturveränderungen sind auch im Bereiche des Höheren Lehramtes denkbar. Allerdings wird die zukünftige Ausbildung der Mittelschullehrer nach wie vor auf die Mitwirkung der Fachinstitute angewiesen sein.

Am Geographischen Institut hat man erkannt, daß der didaktische Auftrag sich nicht nur auf die zukünftigen Lehrer erstrecken soll. Die Geographiedidaktik muß sich in Zukunft zusätzlich folgenden Aufgabenbereichen zuwenden:

1. Ausbau der Fachdidaktik: Ein Ausbau der Fachdidaktik erscheint im Hinblick auf die zukünftigen Aufgaben als notwendig. Von einem vollamtlichen Fachdidaktiker wäre allerdings abzusehen, da eine praxisgestützte Didaktik die Unterrichtstätigkeit des Didaktikers bedingt.

2. Fortbildung: «Lebenslänglich» wird für einen gewählten Lehrer in Zukunft mehr und mehr auch lebenslanges Lernen beinhalten. Das Geographische Institut möchte sich auch in Zukunft diesem Aufgabenkreis widmen (Abb. 2) und dadurch die Beziehung des Institutes zu den Geographielehrern vertiefen. Langjährige Erfahrungen aus vielen Fortbildungskursen für Lehrkräfte verschiedenster Schulstufen sind vorhanden (Liste in VSGg 1986: 6-9). Die Weiterausbildung müßte sich in Zukunft auch auf Geographen im außerschulischen Arbeitsfeld (Industrie, Verwaltung) erstrecken. Damit würde das Geographische Institut weitgehend Neuland betreten.

3. Didaktische Ausbildung: In seiner zukünftigen Tätigkeit sieht sich jeder Geograph mit der Situation konfrontiert, Arbeitsergebnisse, Berichte usw. vor einem breiteren Publikum zu vertreten. Ein didaktischer Grundkurs, der den Studenten schon an der Universität in Seminarien und Kolloquien zugute käme, ist zu entwickeln. Auch dem Mittelbau, der am Geographischen Institut selbständig Lehrveranstaltungen durchzuführen hat, würde dieser Kurs dienen. Da auf Universitätsebene keine entsprechende Didaktik angeboten wird, muß das Institut selbst diese Lücke schließen.

4. Mediendidaktik: Geographische Forschung will präsentiert sein. Andererseits treten dem Studenten häufig geographische Informationen in Medien (Radio, Fernsehen, Film) entgegen. Eine mediendidaktische Einführung muß dem Studenten helfen, mit Medien zu lernen, aber auch Ergebnisse mediengerecht vorzuführen. Da Geographen auch im Medienbereich arbeiten könnten, würde sich damit hier eine erste Kontaktnahme ergeben. (Ein erster Kurs, durchgeführt vom Fachdidaktiker, wird im Winter 1989/90 stattfinden.)

5. Zusammenarbeit mit anderen Universitäten: Die aus einer Arbeitsgruppe 1985 hervorgegangene 


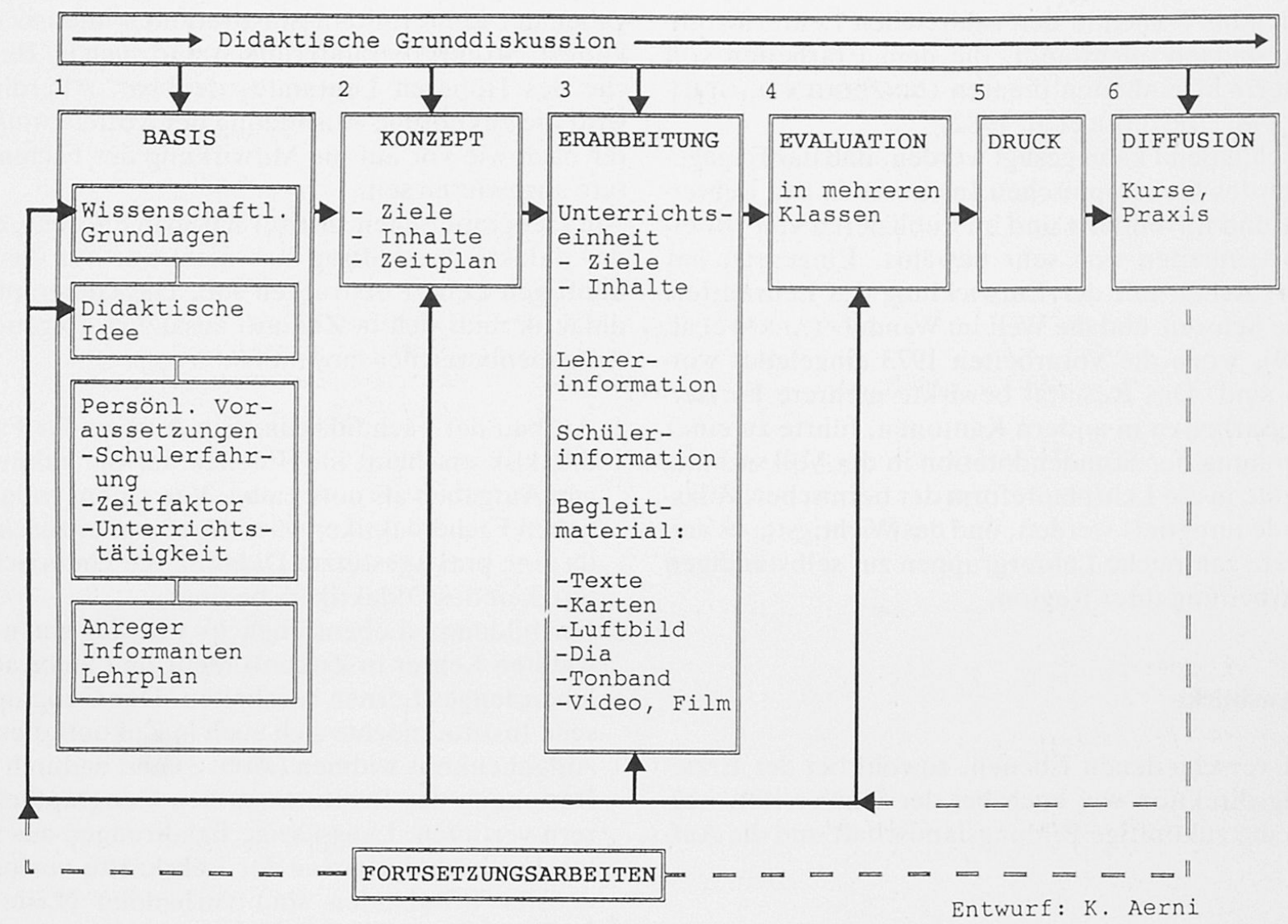

Abb. 5 Konzeptionelles Modell für die Entwicklung geographischer Unterrichtseinheiten auf Stufe Lizentiat oder Doktorat

Geographiedidaktikerkonferenz führt ihre Arbeiten in regelmäßigen Sitzungen weiter. Sie vertritt die Anliegen der Didaktiker gegenüber Institutionen und Behörden (Schweiz. Geogr. Ges., VSGg usw.).

6. Didaktische Forschungsarbeiten: Auf eine eigentliche theoriedidaktische Forschung soll auch in Zukunft verzichtet werden. Es werden jedoch weiterhin praxisgestützte Beiträge und Umsetzungen entwickelt, die an Geographie-Symposien vorgetragen werden. Weitere Schwerpunkte müßten in der Exkursionsdidaktik und in der Mediendidaktik liegen.

\section{Zusammenfassung}

Die Ausbildung von Geographielehrern gehört seit der Gründung des Geographischen Institutes (1886) zu dessen Hauptaufgaben. Das sogenannte «Berner Modell», wie es von auswärtigen Kollegen bezeichnet wird, besitzt folgende Merkmale:

1. Im Dreieckbezug Forschung-Lehre-Praxis wird die Lehrerausbildung als wesentliche Umsetzung und Ausstrahlungsmöglichkeit für die Disziplin wie für das Institut betrachtet. Auf Stufe Lizentiat und Dissertation können daher unter bestimmten Voraussetzungen auch fachdidaktische Themen bearbeitet werden.

2. Zu den verschiedenen Schulstufen und zur Lehrerfortbildung bestehen persönliche, intensive vertikale Kontakte, da die meisten Dozenten seinerzeit selber eine Lehrerausbildung absolviert haben.

3. Der Leiter der Fachdidaktik Geographie ist im Institut integriert. Neben seinem Lehrauftrag betreut er zusätzlich regionalgeographische Kurse und künftighin auch die Mediendidaktik für alle Geographiestudenten.

4. Die konkreten Beiträge des Institutes an die Lehrerfortbildung bestehen in der Durchführung von Kursen und Exkursionen, ferner in der Erarbeitung und Herausgabe von Lehrmitteln und Dokumentationsmaterial sowie in zahlreichen Beratungen.

Wir danken R. Kalbermatten für das Zeichnen der Figuren und G. Pfander für die Durchsicht des Manuskriptes. 


\section{Anmerkungen}

1 Am Seminar Hofwil waren u. a. als Geographielehrer tätig: Fritz Nussbaum, 1914-1944 (Prof. 1922-1949)

Fritz Gygax, 1944-1949 (Prof. 1949-1978)

Georges Grosjean, 1949-1952 (Prof. 1962-1986)

Klaus Aerni, 1961-1974 (Prof. ab 1978)

2 Auslandexkursionen des Bernischen Mittellehrervereins (BMV) unter wissenschaftlicher Leitung des Geogr. Institutes:

1965 Querprofil Skandinavien (B. Messerli, K. Aerni)

1967 Tschechoslowakei - Polen (B. Messerli)

1971 Östliche USA (B. Messerli, K. Aerni)

1973 Westrußland (K. Aerni, Ch. Zürcher)

1975 Kaukasus (K. Aerni, Ch. Zürcher)

1977 Mexiko - Guatemala (K. Aerni, H. Kienholz)

1979 Nordindien, Kaschmir, Afghanistan, Pakistan (K. Aerni, E. Grütter)

1981 Nördliche und mittlere Anden (K. Aerni, E. Grütter)

1985 Südafrika (K. Aerni, E. Grütter)

1987 Sowjetunion: Leningrad-Tiflis (K. Aerni, E. Grütter)

1989 Südamerika (K. Aerni, E. Grütter)

Organisatorische Leitung: P. Michel, Beauftragter des BMV. An den Reisen können sich Lehrkräfte aller Stufen beteiligen.

${ }^{3}$ Blockkurse in Regionalgeographie unter Leitung von Dr. M. Hasler, Lehrbeauftragter für Fachdidaktik:

1986 Der Nahe Osten - Regionaler Konfliktherd im globalen Spannungsfeld (Dr. Arnold Hottinger, Nikosia)

1987 Brasilien - wirtschaftliche Entwicklung und Probleme eines Schwellenlandes. Informationen und didaktische Umsetzung (Dr. Jürg Müller, Schaffhausen)

1988 Japan - Kulturelle Identität und wirtschaftliche Entwicklung - Information und didaktische Umsetzung Dr. Dusan Simko, Basel)

1989 Sowjetunion (Prof. J. Stadelbauer, Mainz)

\section{Literatur}

AERNI, K. et al. (1979): Die Schweiz und die Welt im Wandel. Teil 1: Arbeitshilfen und Lernplanung. Teil 2: Lehrerdokumentation. Bern: Staatlicher Lehrmittelverlag und Geographica Bernensia, Bände S 4 und S 5.
AERNI, K. et al. (1986): Die Rolle der Geographie in der Gesellschaft. In: Geographica Helvetica 41/1986, S. 126-154.

AERNI, K. (Redaktion) (1988): "Geographie im Jahre 2000" Forschungspolitische Früherkennung (FER) für die Geographie. In: Geographica Helvetica 43/1988, 37-44.

BINZEGGER, R. und GRÜTTER, E. (1981): Die Schweiz aus dem All. Einführungspraktikum in das Satellitenbild. Bern: Geographica Bernensia, Heft S 7.

BUFF, E. (1987): Das Berggebiet. Abwanderung, Tourismus regionale Disparitäten. Unterrichtseinheit für die Sekundarstufe II. Bern: Geographica Bernensia, Heft S 12.

GROSJEAN, G. (1986): 100 Jahre Geographie an der Universität Bern 1886-1986. In: Berner Geographische Mitteilungen, 83-92.

GRÜTTER, E. et al. (1982): Landschaftsökologie im Geographieunterricht. Heft 2: Vier geographische Praktikumsaufgaben für Mittelschulen. Bern: Geographica Bernensia, Heft S 9 .

LEIMGRUBER, W. (Redaktion) (1988): Leitbild Geographie Schweiz. In: Geographica Helvetica 43/1988, 33-36.

PFANDER, G. (1983): Exkursionsvorschläge für den Raum des Kantons Bern und Didaktik geographischer Geländearbeit. Bern: Geogr. Institut.

STAB - Staatsarchiv Bern, Universitätsakten 1886.

STUCKI, A. (1984): Vulkan Dritte Welt. 150 Millionen Indonesier blicken in die Zukunft. Unterrichtseinheit für die Sekundarstufe II. Bern: Geographica Bernensia, Heft S 10.

VERDUIN-MULLER, H. (1983): About the identity of Informational Geography for formal, non-formal and informal educational purposes. In: Geography for Education, Paper Book 1974-1984, S. 9-1 bis 9-9. Utrecht: Geographical Institute, University.

VSGg - VEREIN SCHWEIZERISCHER GEOGRAPHIELEHRER, 1986: Schulgeographie in der Schweiz. Eine Standortbestimmung aus Anlaß der 75-Jahr-Feier 1986. Herausgegeben vom VSGg. Trimmis. 